\title{
Efectos de la vacuna neumocócica conjugada (VCN7 y VCN13) en los niños turcos con enfermedad neumocócica invasiva: experiencia en un solo centro
}

\author{
The effects of pneumococcal conjugate vaccine (PCV7 and \\ PCV13) on Turkish children with invasive pneumococcal
}

a. Facultad de Medicina de Ankara Üniversitesi, Departamento de Infectología Pediátrica, Ankara, Turquía.

b. Facultad de Medicina de Ankara Üniversitesi, Departamento de Pediatría, Ankara, Turquía.

c. Agencia de Salud Pública de Turquía, Departamento de Laboratorios de Microbiología de Referencia, Laboratorio Nacional de Investigación y Aplicación de Microbiología Molecular, Ankara, Turquía.

d. Hospital de Formación e Investigación de Okmeydanı, Clínica de Infectología Pediátrica, Estambul, Turquía

e. Hospital de Formación e Investigación de Antalya, Clínica de Infectología Pediátrica, Antalya, Turquía

f. Facultad de Medicina de Ankara Üniversitesi, Laboratorio de Microbiología del Hospital del Campus Cebeci, Ankara, Turquía.

Correspondencia: Dr. Halil Özdemir: doktorhalil@gmail.com

Financiamiento:

Ninguno.

Conflicto de intereses: Ninguno que declarar.

Recibido: 29-9-2016 Aceptado: 16-1-2017

disease: a single center experience

\author{
Dr. Halil Özdemir,${ }^{a}$ Dr. Caner Ylldlz, ${ }^{b}$ Dra. Selin Nar Ötgün, ${ }^{c}$ Dra. Hatice Erkol, ${ }^{b}$ \\ Dr. Adem Karbuz, ${ }^{d}$ Dra. Bilge Aldemir Kocabaş, ${ }^{e}$ Dra. Tuğçe Tural Kara, ${ }^{a}$ \\ Prof. Dra. Ayşsegül Gözalan, ${ }^{c}$ Prof. Dr. Rlza Durmaz, ${ }^{c}$ Prof. Dr. Ergin Çiftçi, \\ Prof. Dr. Derya Aysev y Prof. Dr. Erdal İnce ${ }^{a}$
}

\section{RESUMEN}

Introducción: Nuestro objetivo fue determinar los cambios en la incidencia de enfermedad neumocócica invasiva (ENI), la distribución de serotipos y patrones de resistencia antibiótica del Streptococcus pneumoniae en niños con ENI tras el período de vacunación (de 1 a 7 años) con vacuna neumocócica de 7 serotipos (VCN7) (2008) y de 13 serotipos (VCN13) (2011).

Población y métodos: El estudio se realizó en 39 niños con ENI de 1 mes a 18 años de edad en Angora, Turquía. Se identificó Streptococcus pneumoniae en sangre, líquido cefalorraquídeo, líquido pleural, y otros tejidos y líquidos corporales estériles mediante procedimientos estándar. Se analizó la resistencia de cepas aisladas de S. pneumoniae a penicilina y ceftriaxona con la prueba deepsilometría(E-test).Losserotipos delas cepassedeterminaron conla reacción deQuellung. Resultados:Laincidencia anual deENIdisminuyó significativamente de 7,71 (intervalo de confianza [IC] del 95\%: de 1,99 a 13,4) a 1,58 (IC del 95\%: de 0,6 a 3,77; reducción del riesgo relativo $=-79,5$; $p=0,006$ ) cada 100000 habitantes de $\leq 5$ años de edad sin enfermedad preexistente. Durante todo el período del estudio, los serotipos en la VCN7 y en la VCN13 representaron el $27,8 \%$ y el $63,8 \%$ de las cepas aisladas, respectivamente. Los serotipos en la VCN13 correspondían al 81,8\% de los casos de ENI en la era previa a la introducción de esta vacuna, y disminuyeron al $56 \%$ en los cuatro años posteriores. Las tasas de resistencia a penicilina y ceftriaxona (en el caso de la meningitis) fueron del $48,5 \%$ y el $9,1 \%$, respectivamente.

Conclusiones: Este estudio observó una disminución significativa en la incidencia de ENI después de la introducción de la VCN13. Palabras clave: niño, incidencia, enfermedad neumocócica invasiva, vacuna neumocócica, Streptococcus pneumoniae.

http:/ / dx.doi.org/10.5546/aap.2017.316 Texto completo en inglés:

http:/ / dx.doi.org/10.5546/ aap.2017.eng.316

Cómo citar: Özdemir H, Yıldız C, Nar Ötgün S, et al. Efectos de la vacuna neumocócica conjugada (VCN7 y VCN13) en los niños turcos con enfermedad neumocócica invasiva: experiencia en un solo centro. Arch Argent Pediatr 2017;115(4):316-322.

\section{INTRODUCCIÓN}

El neumococo, Streptococcus pneumoniae, es un microorganismo patógeno importante que produce neumonía, bacteriemia, sepsis y meningitis; todas estas enfermedades causan morbimortalidad significativa en todo el mundo, en particular en los niños menores de 5 años y en las personas de edad avanzada. ${ }^{1} \mathrm{La}$ introducción de la vacuna neumocócica conjugada de 7 serotipos (VCN7) tanto en niños vacunados como en las personas de edad avanzada no vacunadas en los Estados Unidos (EE. UU.) y muchos otros países se asoció con una reducción de la enfermedad neumocócica invasiva (ENI), en especial de los serotipos en la VCN7 dada la disminución en la portación de estos. Sin embargo, el uso de la VCN7 modificó la epidemiología de la enfermedad neumocócica y la colonización; estudios posteriores documentaron un aumento de las tasas de portación e infección causadas por serotipos no incluidos en la VCN7, lo que disminuyó su efecto sobre la incidencia global de ENI. ${ }^{2,3}$

La VCN7 se introdujo en Turquía en septiembre de 2005 y se incluyó en el calendario nacional de vacunación con un cronograma de $3+1$ en noviembre de 2008 para los niños nacidos a partir de mayo de 2008 . En junio de 2011, el Ministerio de Salud de Turquía recomendó la 
vacunación de rutina con la vacuna neumocócica de 13 serotipos (VCN13) en bebés a los 2, 4, 6 y 12 meses de edad para reemplazar la VCN7, sin vacunación de rescate para los niños mayores, excepto aquellos con riesgo elevado de ENI. Nuestro objetivo fue determinar los cambios en la incidencia de ENI, la distribución de serotipos y los patrones de resistencia antibiótica del Streptococcus pneumoniae en niños con ENI tras el período de vacunación (de 1 a 7 años) con la VCN7 y la VCN13.

\section{POBLACIÓN Y MÉTODOS}

Este estudio se llevó a cabo en niños con ENI que asistieron a los consultorios pediátricos generales y la sala de emergencias pediátricas de la Facultad de Medicina de la Ankara Üniversitesi en Angora, Turquía, entre el 1 de septiembre de 2009 y el 30 de septiembre de 2015. En el estudio se incluyó solamente a los niños inmunocompetentes con ENI en los que se hubiera aislado $S$. pneumoniae en sangre y líquidos corporales estériles. Se excluyó de la población del estudio a los niños inmunocomprometidos y a los niños con S. pneumoniae aislado en líquidos corporales o tejidos no estériles. Se definió el estado de inmunización de los niños como con vacunación completa ( 3 o 4 dosis antes de los 24 meses y 1 dosis a los 24 meses o después) y con vacunación parcial ( 1 o 2 dosis antes de los 24 meses) conforme a los criterios del Comité Asesor sobre Prácticas de Inmunización (Advisory Committee on Immunization Practices, ACIP). ${ }^{4}$

Se enviaron las cepas aisladas en sangre, líquido cefalorraquídeo, líquido pleural, y otros tejidos y líquidos corporales estériles dentro de las dos horas al laboratorio de microbiología de la Facultad de Medicina de la Ankara Üniversitesi, donde se procesaron. Las muestras se inocularon en placas de agar suplementadas con sangre ovina desfibrinada al 5\% y se las incubó a $37^{\circ} \mathrm{C}$ en una atmósfera de $\mathrm{CO}_{2}$ entre $5 \%$ y $10 \%$. Se identificó S. pneumoniae mediante procedimientos estándar de laboratorio, tales como la morfología tras la tinción de Gram, la susceptibilidad en disco de optoquina de $5 \mu \mathrm{g}$ y la prueba de solubilidad de la bilis.

Se analizó la resistencia de las cepas de S. pneumoniae aisladas a la penicilina y la ceftriaxona con la prueba de epsilometría (E-test). Se usó S. pneumoniae ATCC 49619 en antibiogramas. ${ }^{5}$ Los valores críticos y las concentraciones inhibitorias mínimas (CIM) se interpretaron de acuerdo con las recomendaciones de 2008 del Instituto de Normas Clínicas y de Laboratorio (Clinical Laboratory Standards Institute, CLSI). ${ }^{6}$ Brevemente, los criterios para definir meningitis en relación con la penicilina fueron los siguientes: susceptible $(\mathrm{CIM} \leq 0,06 \mu \mathrm{g} / \mathrm{ml})$ o con alto nivel de resistencia $(\mathrm{CIM} \geq 0,12 \mu \mathrm{g} / \mathrm{ml})$. Los criterios para establecer la ausencia de meningitis (uso parenteral) en relación con la penicilina fueron los siguientes: susceptible (CIM $\leq 2 \mu \mathrm{g} / \mathrm{ml})$, con bajo nivel de resistencia (CIM de 2 a $4 \mu \mathrm{g} / \mathrm{ml}$ ) y con alto nivel de resistencia (CIM $\geq 8 \mu \mathrm{g} / \mathrm{ml})$; en tanto, los criterios para establecer la ausencia de meningitis (uso oral) en relación con la penicilina fueron los siguientes: susceptible $(\mathrm{CIM} \leq 0,06 \mu \mathrm{g} / \mathrm{ml})$, con bajo nivel de resistencia (CIM de 0,12 a 1,0 $\mu \mathrm{g} / \mathrm{ml}$ ) y con alto nivel de resistencia $(\mathrm{CIM} \geq 2 \mu \mathrm{g} / \mathrm{ml})$. Los criterios para definir meningitis en relación con la ceftriaxona fueron los siguientes: susceptible $(\mathrm{CIM} \leq 0,5 \mu \mathrm{g} / \mathrm{ml})$, con bajo nivel de resistencia $(\mathrm{CIM} \geq 1,0 \mu \mathrm{g} / \mathrm{ml})$ y con alto nivel de resistencia $(\mathrm{CIM} \geq 2 \mu \mathrm{g} / \mathrm{ml})$. Los criterios para definir la ausencia de meningitis en relación con la ceftriaxona fueron los siguientes: susceptible $(\mathrm{CIM} \leq 1 \mu \mathrm{g} / \mathrm{ml})$, con bajo nivel de resistencia (CIM $=2 \mu \mathrm{g} / \mathrm{ml}$ ) y con alto nivel de resistencia (CIM $\geq 4 \mu \mathrm{g} / \mathrm{ml})$. Las cepas aisladas con una CIM mayor al valor crítico para establecer la susceptibilidad se indicaron como "no susceptibles", es decir, como cepas con bajo y alto nivel de resistencia. Entre las cepas aisladas en el estudio, $33(84,6 \%)$ se evaluaron con antibiogramas.

Después de completar los antibiogramas, se enviaron las cepas aisladas a la Agencia de Salud Pública de Turquía, Departamento de Laboratorios de Microbiología de Referencia, Laboratorio Nacional de Investigación y Aplicación de Microbiología Molecular, Angora, Turquía en un medio de transporte Amies con carbón; las cepas se conservaron congeladas en citrato de glicerol a $-80^{\circ} \mathrm{C}$. Los serotipos se determinaron mediante la reacción de Quellung con antisueros obtenidos del Statens Serum Institut (Copenhague, Dinamarca). De las cepas aisladas incluidas en el estudio, se estableció el serotipo de 36 (92,3\%).

Se obtuvo el consentimiento informado de los padres; el estudio fue aprobado por el comité de ética de la Facultad de Medicina de la Ankara Üniversitesi. Todas las pruebas estadísticas se completaron con el paquete estadístico SPSS. Se calculó la incidencia de ENI (100 000/pacientes ambulatorios admitidos) según la proporción entre cantidad de niños con ENI y cantidad de niños que asisten a los consultorios pediátricos generales y la sala de emergencias pediátricas de 
nuestro hospital en el mismo período. Asimismo, solo se consideró a los niños de $\leq 5$ años para calcular la incidencia. El cambio en la tasa de incidencia entre el primer y el último año del estudio se evaluó estimando la reducción del riesgo absoluto (RRA) y la reducción del riesgo relativo (RRR). Las diferencias se consideraron significativas si el valor de $p$ era $<0,05$.

TABLA 1. Características clínicas de los niños con ENI, distribución de serotipos y patrones de resistencia antibiótica de las cepas aisladas

\begin{tabular}{|c|c|c|c|c|c|c|c|c|c|}
\hline \multirow{2}{*}{$\begin{array}{l}\text { Período } \\
\text { VCN7 }\end{array}$} & \multirow{2}{*}{$\begin{array}{c}\text { Edad } \\
\text { (meses) }\end{array}$} & \multirow{2}{*}{$\begin{array}{c}\text { Sexo } \\
\mathrm{F}\end{array}$} & \multirow{2}{*}{$\begin{array}{c}\text { Diagnóstico } \\
\text { Bacteriemia }\end{array}$} & \multirow{2}{*}{$\begin{array}{c}\begin{array}{c}\text { Sitio de } \\
\text { obtención }\end{array} \\
\text { Sangre }\end{array}$} & \multirow{2}{*}{$\begin{array}{c}\text { Vacunación } \\
\text { VCN7 }\end{array}$} & \multirow{2}{*}{$\begin{array}{l}\text { Enfermedad } \\
\text { preexistente }\end{array}$} & \multirow{2}{*}{$\begin{array}{c}\begin{array}{c}\text { Susceptibilidad } \\
\text { a la penicilina } \\
\text { (CIM) }\end{array} \\
0,006\end{array}$} & \multicolumn{2}{|c|}{$\begin{array}{l}\text { Susceptibilidad Serotipo } \\
\text { a la ceftriaxona } \\
\text { (CIM) }\end{array}$} \\
\hline & & & & & & & & 0,008 & $7 \mathrm{~F}$ \\
\hline & 34 & $\mathrm{~F}$ & Bacteriemia & Sangre & NV & & 2 & 1 & $23 \mathrm{~F}$ \\
\hline & 27 & M & $\begin{array}{l}\text { Mastoiditis- } \\
\text { y absceso } \\
\text { subperióstico }\end{array}$ & Absceso & NV & & 0,125 & 0,125 & $19 \mathrm{~F}$ \\
\hline & 38 & $\mathrm{~F}$ & Bacteriemia & Sangre & NV & & 0,016 & 0,047 & $18 \mathrm{~F}$ \\
\hline & 6 & M & Bacteriemia & Sangre & PV & & 0,19 & 0,19 & 14 \\
\hline & 15 & $\mathrm{~F}$ & Meningitis & LCR-Sangre & VCN7 & & 0,016 & 0,002 & 10 \\
\hline & 40 & F & Meningitis & LCR-Sangre & NV & & 0,19 & 0,19 & $6 \mathrm{~A}$ \\
\hline & 110 & M & Meningitis & LCR & NV & & 1 & 0,50 & $23 \mathrm{~F}$ \\
\hline & 4 & $\mathrm{~F}$ & Bacteriemia & Sangre & PV & & 0,016 & 0,008 & $7 \mathrm{~F}$ \\
\hline & 93 & M & Neumonía & Sangre & NV & & No definido & No definido & No definido \\
\hline & 11 & M & Meningitis & LCR & VCN7 & & 0,64 & 0,25 & $19 \mathrm{~A}$ \\
\hline & 3 & $\mathrm{~F}$ & Bacteriemia & Sangre & PV & & 0,5 & 0,38 & $19 \mathrm{~F}$ \\
\hline \multirow[t]{27}{*}{ VCN13 } & 24 & M & Bacteriemia & Sangre & VCN7 & & 0,047 & 0,023 & $15 \mathrm{~F} / \mathrm{A} / \mathrm{B} / \mathrm{C}$ \\
\hline & 9 & M & Bacteriemia & Sangre & VCN7 & & No definido & No definido & No definido \\
\hline & 34 & F & $\begin{array}{l}\text { Celulitis } \\
\text { periorbital }\end{array}$ & Sangre & VCN7 & & 0,016 & 0,047 & $17 \mathrm{~F} / \mathrm{A}$ \\
\hline & 33 & M & Bacteriemia & Sangre & VCN7 & & 0,190 & 0,190 & $19 \mathrm{~F}$ \\
\hline & 13 & M & Neumonía & Sangre & VCN7 & & $<0,016$ & 0,023 & 8 \\
\hline & 71 & M & Bacteriemia & Sangre & NV & Hemofilia A & No definido & No definido & No definido \\
\hline & 24 & M & Bacteriemia & Sangre & VCN7 & & 0,75 & 0,25 & $35 \mathrm{~B}$ \\
\hline & 117 & M & Peritonitis & Sangre & NV & $\begin{array}{l}\text { Hepatopatía } \\
\text { crónica }\end{array}$ & 0,094 & 0,190 & 8 \\
\hline & 1 & F & Bacteriemia & Sangre & NV & & $<0,016$ & 0,008 & $7 \mathrm{~F}$ \\
\hline & 16 & M & Bacteriemia & Sangre & VCN13 & & 1,5 & 1 & $15 \mathrm{~F} / \mathrm{A}$ \\
\hline & 2 & $\mathrm{~F}$ & Piomiositis & Sangre & PV & & $<0,016$ & 0,032 & 5 \\
\hline & 93 & $\mathrm{~F}$ & Peritonitis & $\begin{array}{l}\text { Líquido } \\
\text { peritoneal }\end{array}$ & NV & $\begin{array}{l}\text { Síndrome } \\
\text { nefrótico }\end{array}$ & 0,094 & 0,064 & $6 \mathrm{~A}$ \\
\hline & 19 & $\mathrm{~F}$ & Bacteriemia & Sangre & VCN13 & & $<0,016$ & 0,008 & $33 \mathrm{~F} / \mathrm{A} / \mathrm{B} / \mathrm{C} / \mathrm{D}$ \\
\hline & 198 & M & Meningitis & LCR & NV & & No definido & No definido & $23 \mathrm{~F}$ \\
\hline & 8 & M & Bacteriemia & Sangre & VCN13 & & 1,5 & 0,38 & $19 \mathrm{~F}$ \\
\hline & 216 & $\mathrm{~F}$ & Bacteriemia & Sangre & $\operatorname{VCN}^{13} \underset{(\mathrm{p}}{\mathrm{T}}$ & $\begin{array}{l}\text { Talasemia mayor } \\
\text { posterior a TMO) }\end{array}$ & 1 & 0,75 & $23 \mathrm{~F}$ \\
\hline & 68 & M & Neumonía & Sangre & NV & & 0,016 & 0,012 & 1 \\
\hline & 13 & F & Bacteriemia & Sangre & VCN13 & & 0,064 & 0,016 & $15 B / C$ \\
\hline & 4 & M & Meningitis & LCR & PV & & No definido & No definido & $35 \mathrm{~A} / \mathrm{B} / \mathrm{C}$ \\
\hline & 40 & $\mathrm{~F}$ & $\begin{array}{l}\text { Mastoiditis- } \\
\text { y absceso } \\
\text { subperióstico }\end{array}$ & Absceso & VCN13 & & 1,5 & 0,5 & $19 \mathrm{~F}$ \\
\hline & 120 & M & Meningitis & LCR & NV & $\begin{array}{l}\text { Posterior a } \\
\text { traumatismo }\end{array}$ & No definido & No definido & $6 \mathrm{~A}$ \\
\hline & 4 & M & Bacteriemia & Sangre & PV & & $<0,016$ & 0,008 & $12 \mathrm{~B}$ \\
\hline & 32 & M & Neumonía & Sangre & VCN13 & & $<0,016$ & 0,004 & 3 \\
\hline & 129 & M & Neumonía & Sangre & NV & & $<0,016$ & 0,008 & $7 \mathrm{~F}$ \\
\hline & 39 & M & Bacteriemia & Sangre & VCN13 & $\begin{array}{l}\text { Leucemia } \\
\text { linfocítica } \\
\text { aguda }\end{array}$ & 0,064 & 0,016 & 3 \\
\hline & 36 & M & Neumonía & Líquido pleura & alVCN13 & & 0,75 & 0,5 & $19 \mathrm{~A}$ \\
\hline & 46 & $\mathrm{~F}$ & Meningitis & LCR-Sangre & VCN13 & $\begin{array}{l}\text { Posterior a } \\
\text { traumatismo }\end{array}$ & 0,047 & 0,047 & 21 \\
\hline
\end{tabular}

NV: no vacunado. PV: parcialmente vacunado. TMO: trasplante de médula ósea. 


\section{RESULTADOS}

El estudio incluyó 39 niños con ENI en el Hospital Pediátrico de la Ankara Üniversitesi. La proporción entre varones y mujeres era 1,29. La mediana de edad de los niños era 32 meses (1 mes-18 años); 29 (74,4\%) eran menores de 5. El diagnóstico final de los pacientes fue bacteriemia (19/39), meningitis (8/39), neumonía $(6 / 39)$, mastoiditis y absceso subperióstico $(2 / 39)$, peritonitis (2/39), celulitis periorbital (1/39) y piomiositis (1/39). La mayoría de las cepas aisladas de S. pneumoniae $(69,2 \%)$ se hallaron en la sangre. Entre los niños incluidos en el estudio, $19(48,7 \%)$ estaban completamente vacunados (9/19 con la VCN7 y 10/20 con la VCN13); los 20 niños restantes $(51,3 \%)$ estaban en el grupo parcialmente vacunado y no vacunado. En la Tabla 1, se indican las características clínicas de los niños con ENI, la distribución de serotipos y los patrones de resistencia antibiótica de las cepas aisladas.

De acuerdo con las CIM de las cepas aisladas, la tasa de resistencia a la penicilina (administrada por vía oral y parenteral para la meningitis) fue del 48,5\% (16/33), pero en relación con la administración parenteral en los casos no definidos como meningitis, la tasa de resistencia a la penicilina fue del 3,3\% (1/33). Las tasas de resistencia a la ceftriaxona en los casos de meningitis y ausencia de meningitis fueron del $9,1 \%(3 / 33)$ y $0 \%$, respectivamente.

Durante todo el período del estudio, los serotipos en la VCN7 y la VCN13 representaron el $27,8 \%(10 / 36)$ y el $63,8 \%(23 / 36)$ de las cepas aisladas, respectivamente. Los serotipos en la VCN13 correspondieron al 81,8\% de los pacientes $(9 / 11)$ con ENI en la era previa a su introducción, y disminuyeron al 56\% (14/25) en los cuatro años posteriores. De manera similar, el porcentaje de serotipos en la VCN7 en los pacientes con ENI disminuyó del 45,5\% (5/11) al 20\% (5/25) en el mismo período. Durante el primer período (la era de la VCN7), ninguna de las cepas aisladas correspondía a los serotipos en la VCN7 en los niños vacunados, y dos de los tres serotipos no incluidos en la VCN7 (serotipos 7F y 19A) estaban cubiertos por la VCN13. Por otro lado, la distribución de los serotipos en los niños parcialmente vacunados y no vacunados fue la siguiente: 5 eran serotipos en la VCN (serotipo 19F: 2, serotipo 23F: 2 y serotipo 14: 1), 3 eran serotipos no incluidos en la VCN7, aunque 2 de ellos estaban cubiertos por la VCN13 (serotipos 6A y 7F) en el mismo período. Durante el segundo período (la era de la VCN13), 1 de las 5 cepas aisladas correspondía a un serotipo de la VCN7 (serotipo 19F) y ninguno de los serotipos no incluidos en la VCN7 estaban cubiertos por la VCN13 en los niños vacunados con la VCN7. Sin embargo, la cantidad de serotipos en la VCN13 fue de 6 en 10 niños vacunados con la VCN13 (serotipo 19F: 2, serotipo 3: 2, serotipo 23F: 1 y serotipo 19A: 1) y de 7 en 10 niños no vacunados (serotipo 7F: 2, serotipo 6A: 2, serotipo 5: 1 , serotipo 23F: 1 y serotipo 1: 1) en el mismo período.

La incidencia anual general de ENI disminuyó significativamente de 7,71 (intervalo de confianza [IC] del 95\%: 1,99-13,4) a 1,58 (IC del 95\%: 0,6-3,77; $\mathrm{RRR}=-79,5 ; p=0,006)$ cada 100000 habitantes de $\leq 5$ años de edad sin enfermedad subyacente. La disminución en la incidencia de ENI se tornó más evidente tras la implementación de la VCN13 (Figura 1).

FIGURA 1. Incidencia anual de ENI en el Hospital Pediátrico de la Ankara Üniversitesi

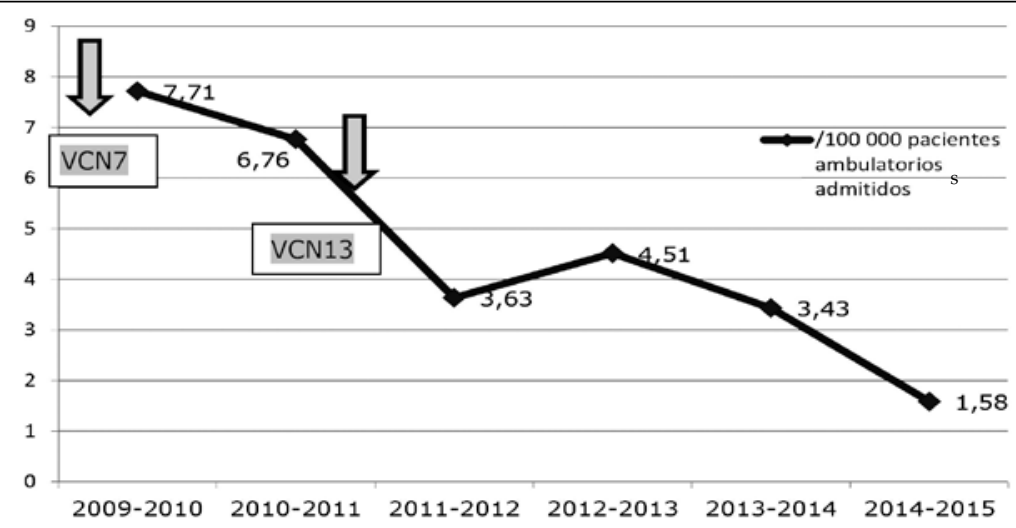




\section{DISCUSIÓN}

Este es el primer estudio en Turquía sobre el cambio en patrones de incidencia de ENI en niños turcos después de la inclusión de la VCN7 y la VCN13 en el calendario nacional de vacunación La vacunación de los niños turcos con $\mathrm{VCN}$ ha sido eficaz y continua durante siete años. No obstante, en este país, la información sobre los efectos de la VCN7 y la VCN13 en niños con ENI sigue siendo insuficiente. Se realizaron algunos estudios sobre la distribución de los serotipos y los patrones de resistencia antibiótica de los neumococos tras la introducción de la VCN7 y la VCN13. Sin embargo, estos estudios no mencionan las modificaciones en la incidencia de ENI pediátrica después de la implementación de las VCN. Si bien en este estudio se incluyó a una cantidad limitada de pacientes, resulta muy valioso para Turquía porque brindará los primeros datos sobre la modificación del patrón de incidencia de ENI en los niños turcos tras la implementación de la VCN7 y la VCN13. Además, se compararon la susceptibilidad antibiótica y el serotipo de las cepas aisladas en este país con los resultados de otros países antes y después de la introducción de las $\mathrm{VCN}$.

Después de la introducción de la VCN7, se observaron disminuciones drásticas en la incidencia de ENI en los niños estadounidenses menores de 5 años. Tales disminuciones fueron el resultado de la reducción de casos provocados por los serotipos en la VCN7. Sin embargo, los casos de ENI causada por los serotipos no incluidos en la VCN7 aumentaron; el serotipo 19A fue el que tuvo la mayor incidencia de ENI en la era posterior a la VCN. Luego, los logros alcanzados en la reducción de la incidencia de ENI se frenaron. Entonces, la VCN13 reemplazó la VCN7 y se utilizó ampliamente en los EE. UU.,8 Farnham y col. informaron que la incidencia de ENI se redujo $69,6 \%$, de 21,0 casos cada 100000 niños menores de 5 antes de la era de la VCN13 a 6,4 casos cada 100000 en la era posterior a la VCN13 en la ciudad de Nueva York, EE. UU. La ENI causada por los serotipos en la VCN13 disminuyeron $82,5 \%$, lo que incluye una reducción de $\sim 80 \%$ en el serotipo 19A; las tasas de cobertura de los serotipos en la VCN13 fueron del $72,9 \%$ y el $42,1 \%$ en la era previa y posterior a la VCN13, respectivamente. ${ }^{9}$ Se obtuvieron resultados similares de estudios realizados en los mismos períodos en Massachusetts y Alaska, EE. UU. En estos estudios, se observó una disminución estadísticamente significativa en las tasas de ENI y de ENI causada por serotipos relacionados con la VCN13, en especial los serotipos 19A, 7F y 3 después de la introducción de la VCN13. De este modo, la tasa general de ENI y de ENI causada por los serotipos en la VCN13 disminuyó 58\% y 83\%, respectivamente, en Alaska. ${ }^{8,10}$ Por otro lado, la cantidad de casos de meningitis neumocócica por año siguió siendo la misma en los niños estadounidenses durante la era de la VCN13. La proporción de serotipos en la VCN13 disminuyó del 54\% al 27\%. Los serotipos más frecuentes cambiaron de 19A, 7F y 3 a 19A, 35B y 22F. La tasa de susceptibilidad a la penicilina fue similar (75\% frente a $75 \%$ ), aunque la tasa de cepas no susceptibles a la ceftriaxona disminuyó significativamente del $13 \%$ al 3\%. ${ }^{11}$

La incidencia de ENI, la distribución de serotipos y la susceptibilidad antibiótica de los neumococos también se vieron afectadas positivamente por las VCN en los países europeos. La incidencia general de ENI en todos los grupos etarios en comparación con la era previa a la VCN7 y previa a la VCN13 en Inglaterra y Gales fue del $56 \%$ y $32 \%$, respectivamente. Pero se observó evidencia de un aumento de ENI causada por los serotipos no incluidos en la VCN13, en particular en niños menores de 5 años en el último año; estos eran los serotipos 8, 15A, 15B/C, 22F, 23B y 24F. ${ }^{3}$ La influencia de la VCN13 en la incidencia de ENI fue prominente en los niños daneses menores de 2 años, con una reducción del $71 \%$, y la incidencia de los 6 serotipos adicionales de la VCN13 se redujo aproximadamente un $84 \%$. Por otro lado, el reemplazo de serotipos se hizo evidente, y casi el $80 \%$ de las causas de ENI del año pasado correspondían a serotipos no incluidos en la $\operatorname{VCN13}(8,10 \mathrm{~A} / \mathrm{B}, 12 \mathrm{~F}, 15 \mathrm{~B} / \mathrm{C}, 20,22 \mathrm{~F}, 33 \mathrm{~F}, 38,23 \mathrm{~B}$, 24F). ${ }^{12}$ De manera similar, se observó el cambio de serotipos en los niños alemanes. La proporción de serotipos en la VCN7 entre las cepas aisladas de los pacientes con ENI disminuyó del 61,8\% al 5,2\%, y el porcentaje de serotipos no incluidos en la VCN13 aumentó del 15,6\% al 59,2\%. Estos serotipos no incluidos en la VCN13 eran 10A, 12F, 23B, 24F y $38 .{ }^{13}$ Se evaluó el impacto positivo importante de la VCN13 sobre la incidencia de meningitis neumocócica en niños franceses menores de 2 años. La disminución de los casos a causa de los serotipos en la VCN7 y los 6 serotipos adicionales de la VCN13 fue del 90,3\% y el 67\%, respectivamente. Los serotipos no incluidos en la VCN13 permanecieron estables hasta el último año del estudio, pero estos representaron el $67,6 \%$ de los casos, principalmente causados por los serotipos $12 \mathrm{~F}$ y $24 \mathrm{~F}$. El 39,5\% de las cepas aisladas no fueron susceptibles a la penicilina, y el $88 \%$ fueron susceptibles a la cefotaxima/ceftriaxona a lo largo de todo el período. ${ }^{14,15}$ 
Se observaron los efectos positivos de las VCN sobre la incidencia de ENI en dos países africanos: Sudáfrica y Marruecos. Las tasas en los niños menores de 2 años disminuyeron de 54,8 casos a 17,0 casos cada 100000 añospersona respecto del período previo a la VCN7, incluida una reducción del $89 \%$ en los casos de ENI a causa de los serotipos en la VCN7 en Sudáfrica. ${ }^{16}$ De manera similar, la incidencia anual general de ENI se redujo significativamente $(60,9 \%)$ en los niños menores de 2 años con la implementación de las VCN en Marruecos. La incidencia general de ENI a causa de serotipos en la VCN7, en la VCN10 pero no en la VCN7 y en la VCN13 pero no en la VCN10 se redujo un $74,1 \%, 77,7 \%$ y $85,2 \%$, respectivamente. Los principales serotipos causantes de ENI en niños menores de 2 años fueron 14, 6B, 19A, 19F, 23F y 5 antes de la vacunación. Solo los serotipos 6B, 14 y 1 persistieron después de esta. Asimismo, las tasas de cepas aisladas no susceptibles a la penicilina disminuyeron del $50,6 \%$ al $21,9 \% .{ }^{17}$ En los niños mexicanos, se observó una disminución significativa en todos los serotipos en la VCN7, del $59,7 \%$ al $21 \%$, y se detectó un aumento gradual del serotipo 19A, del 7\% al 39\%. ${ }^{18}$ Sin embargo, después de la implementación de la VCN13, la incidencia general de ENI se redujo un $75 \%$ y no se informaron casos del serotipo 19A. ${ }^{19}$

En una revisión sistemática sobre el impacto y la eficacia de la VCN10 y la VCN13 sobre la hospitalización y la mortalidad de los niños menores de 5 años en países de América Latina (Brasil, Chile, Uruguay, Argentina, Perú y Nicaragua), la tasas de hospitalización por neumonía, meningitis y ENI confirmadas mediante radiografía disminuyeron $8,8-37,8 \%$, $13,3-87,7 \%$ y $56-83,3 \%$, respectivamente. ${ }^{20}$ Andrade y col. demostraron un impacto significativo de la VCN10 sobre la ENI en los grupos etarios destinatarios de la vacuna en Brasil. Informaron que los serotipos en la VCN10 disminuyeron un $41,3 \%$ y que la ENI en los niños de 2 a 23 meses de edad se redujo un $44,2 \% .{ }^{21}$ De manera similar, un año después de la inclusión de la VCN7 en el calendario de vacunación habitual de Uruguay, se observó una reducción rápida y significativa de las tasas de neumonía extrahospitalaria, neumonía extrahospitalaria neumocócica y meningitis neumocócica del $56 \%$, $48,2 \%$ y $59 \%$, respectivamente..$^{22}$ Además, se determinó una reducción más significativa en las tasas de neumonía extrahospitalaria y neumonía extrahospitalaria neumocócica del 78,1\% y 92,4\%, respectivamente, tras la implementación de la VCN7 y la VCN13 en un período de 9 años. ${ }^{23}$

En Turquía, los estudios sobre la distribución de los serotipos y la susceptibilidad antibiótica de la ENI en niños han sido limitados. Antes de la implementación de las VCN, entre 2001 y 2004, Yalçın y col. informaron que las tasas de cobertura de los serotipos eran del $52 \%, 74 \%$ y $81 \%$ con la VCN7, VCN10 y VCN13, respectivamente. El 39\% y el $14 \%$ de las cepas aisladas no eran susceptibles a la penicilina y la ceftriaxona, respectivamente. ${ }^{24}$ Luego se observaron resultados similares en otros dos estudios sobre meningitis neumocócica en niños; las tasas de cobertura de los serotipos fueron del $52 \%$ frente a $48,1 \%, 74 \%$ frente a $85,2 \%$ y $81 \%$ frente a $92,6 \%$, respectivamente, con la VCN7, VCN10 y VCN13. Los serotipos más frecuentes fueron 1, 5, 6A/B, 19F y $23 \mathrm{~F} .{ }^{25,26} \mathrm{Las}$ VCN mostraron, en primer lugar, sus efectos sobre el reemplazo de serotipos en la colonización nasofaríngea; este fenómeno se mantuvo durante los tres años posteriores a la introducción de la VCN7 en el calendario de vacunación nacional de Turquía en un estudio previo realizado en niños sanos. Las tasas de serotipos cubiertos por la VCN7 y la VCN13 fueron del 46,2\% y el $62 \%$, respectivamente. ${ }^{27} \mathrm{El}$ aumento de los serotipos aislados en la colonización nasofaríngea no incluidos en las vacunas influyen sobre la distribución de las cepas causantes de ENI. En este estudio, los serotipos en la VCN7 y la VCN13 representaron el $27,8 \%$ y el $63,8 \%$ de las cepas aisladas, respectivamente. Los serotipos en la VCN13 correspondían al 81,8\% de los casos de ENI en la era previa a la introducción de esta vacuna, y disminuyeron al $56 \%$ en los cuatro años posteriores. De manera similar, el porcentaje de serotipos en la VCN7 en los pacientes con ENI disminuyó del $45,5 \%$ al $20 \%$ en el mismo período. Asimismo, Ceyhan y col. demostraron que la cobertura potencial de los serotipos variaba entre el $57,5 \%$ y el $36,8 \%$ y entre el $77,4 \%$ y el $60,5 \%$ con la VCN7 y la VCN13 en el período 2008-2014 (era previa a la VCN7 y posterior a la VCN13) en los niños turcos menores de $\leq 5$ años, respectivamente. También hallaron que el porcentaje de serotipos no incluidos en la VCN13 era 37,6 en la era de la VCN13; en nuestro estudio, los serotipos no incluidos en la VCN13 representaron el $44 \%$ en la era de la VCN13. ${ }^{28}$ Si bien el serotipo 19A fue el mayor causante de ENI en niños de los EE. UU y los países europeos en la era posterior a la VCN7, resultó sorprendente que solamente uno de nuestros pacientes de la era posterior a la VCN7 y durante la era de la VCN13 tuviera el serotipo 19A. Además, el serotipo 19A era poco frecuente en los niños turcos con ENI, entre el 1,8\% y el 5,6\%. ${ }^{24-}$ ${ }^{26,28}$ Por otro lado, se identificó el serotipo $19 \mathrm{~F}$ en 3 niños que habían recibido la vacunación completa con VCN7 o VCN13. 


\section{CONCLUSIONES}

A modo de conclusión, este es el primer estudio sobre la modificación en los patrones de incidencia de ENI en niños turcos después de la inclusión de la VCN7 y la VCN13 en el calendario de vacunación nacional de Turquía; se observó una disminución prominente en la incidencia de ENI después de la introducción de la VCN13.

\section{REFERENCIAS}

1. Park M, Kim HS, Shin KS, et al. Changes in the incidence of Streptococcus pneumoniae bacteremia and its serotypes over 10 years in one hospital in South Korea. Vaccine 2014;32(48):6403-7.

2. Mendes RE, Costello AJ, Jacobs MR, et al. Serotype distribution and antimicrobial susceptibility of USA Streptococcus pneumoniae isolates collected prior to and post introduction of 13-valent pneumococcal conjugate vaccine. Diagn Microbiol Infect Dis 2014;80(1):19-25.

3. Waight PA, Andrews NJ, Ladhani SN, et al. Effect of the 13-valent pneumococcal conjugate vaccine on invasive pneumococcal disease in England and Wales 4 years after its introduction: an observational cohort study. Lancet Infect Dis 2015;15(5):535-43.

4. Centers for Disease Control and Prevention (CDC), Advisory Committee on Immunization Practices. Updated recommendation from the Advisory Committee on Immunization Practices (ACIP) for use of 7-valent pneumococcal conjugate vaccine (PCV7) in children aged 24-59 months who are not completely vaccinated. MMWR Recomm Rep 2008;57(13):343-4.

5. Centers for Disease Control and Prevention (CDC). Laboratory methods for the diagnosis of meningitis caused by Neisseria meningitidis, Streptococcus pneumoniae, and Haemophilus influenzae. WHO manual. 2nd edn. Atlanta,GA: WHO Press; 2011:1-14. [Consulta: 17 de enero de 2017] Disponible en: https://www.cdc.gov/meningitis/labmanual/full-manual.pdf

6. Clinical and Laboratory Standards Institute. Performance standards for antimicrobial susceptibility testing; 19th informational supplement. M100-S19. Wayne, PA: Clinical and Laboratory Standards Institute; 2009.

7. Wenger JD, Zulz T, Bruden D, et al. Invasive pneumococcal disease in Alaskan children: impact of the seven-valent pneumococcal conjugate vaccine and the role of water supply. Pediatr Infect Dis J 2010;29(3):251-6.

8. Bruce $M G$, Singleton R, Bulkow L, et al. Impact of the 13-valent pneumococcal conjugate vaccine (pcv13) on invasive pneumococcal disease and carriage in Alaska. Vaccine 2015;33(38):4813-9.

9. Farnham AC, Zimmerman CM, Papadouka V, et al. Invasive pneumococcal disease following the introduction of 13-valent conjugate vaccine in children in New York City from 2007 to 2012. JAMA Pediatr 2015;169(7):646-52.

10. Iroh Tam PY, Madoff LC, Coombes B, et al. Invasive pneumococcal disease after implementation of 13-valent conjugate vaccine. Pediatrics 2014;134(2):210-7.

11. OlarteL, Barson WJ, Barson RM, etal. Impact of the 13-valent pneumococcal conjugate vaccine on pneumococcal meningitis in US children. Clin Infect Dis 2015;61(5):767-75.

12. Harboe ZB, Dalby $T$, Weinberger DM, et al. Impact of 13-valent pneumococcal conjugate vaccination in invasive pneumococcal disease incidence and mortality. Clin Infect Dis 2014;59(8):1066-73.

13. Van der Linden M, Falkenhorst G, Perniciaro S, et al. Effects of infant pneumococcal conjugate vaccination on serotype distribution in invasive pneumococcal disease among children and adults in Germany. PLoS One 2015;10(7):e0131494.

14. Chapoutot AG, Dessein R, Guilluy O, et al. Impact of the 13-valent pneumococcal conjugate vaccine on the incidence of pneumococcal meningitis in children. Epidemiol Infect 2016;144(3):607-11.

15. Levy C, Varon E, Picard C, et al. Trends of pneumococcal meningitis in children after introduction of the 13-valent pneumococcal conjugate vaccine in France. Pediatr Infect Dis J 2014;33(12):1216-21.

16. von Gottberg A, de Gouveia L, Tempia S, et al. Effects of vaccination on invasive pneumococcal disease in South Africa. N Engl J Med 2014;371(20):1889-99.

17. Diawara I, Zerouali $K, K a t f y K$, et al. Invasive pneumococcal disease among children younger than 5 years of age before and after introduction of pneumococcal conjugate vaccine in Casablanca, Morocco. Int J Infect Dis 2015;40:95-101.

18. Echaniz-Aviles G, Soto-Nogueron A, Miranda-Novales $\mathrm{G}$, et al. Streptococcus pneumoniae serotypes identified in Mexican children with invasive disease before and after the introduction of PCV7 (1993-2012). Arch Med Res 2015;46(2):149-53.

19. Chacon-CruzE, Rivas-Landeros RM, Volker-Soberanes ML. Early trends in invasive pneumococcal disease in children following the introduction of 13-valent pneumococcal conjugate vaccine: results from eight years of active surveillance in a Mexican hospital. Ther Adv Vaccines 2014; 2(6):155-8.

20. deOliveira LH, Camacho LA, Coutinho ES, et al. Impact and effectiveness of 10 and 13-valent pneumococcal conjugate vaccines on hospitalization and mortality in children aged less than 5 years in Latin American countries: a systematic review. PLoS One 2016;11(12):e0166736.

21. Andrade AL, Minamisava R, Policena G, et al. Evaluating the impact of PCV-10 on invasive pneumococcal disease in Brazil: a time-series analysis. Hum Vaccin Immunother 2016;12(2):285-92.

22. Pírez MC, Algorta G, Cedrés A, et al. Impact of universal pneumococcal vaccination on hospitalizations for pneumonia and meningitis in children in Montevideo, Uruguay. Pediatr Infect Dis J 2011;30(8):669-74.

23. Pírez MC, Algorta G, Chamorro F, et al. Changes in hospitalizations for pneumonia after universal vaccination with pneumococcal conjugate vaccines $7 / 13$ valent and Haemophilus influenzae type $\mathrm{b}$ conjugate vaccine in $\mathrm{a}$ pediatric referral hospital in Uruguay. Pediatr Infect Dis J 2014;33(7):753-9.

24. Yalçın I, Gürler N, Alhan E, et al. Serotype distribution and antibiotic susceptibility of invasive Streptococcus pneumoniae disease isolates from children in Turkey, 2001-2004. Eur J Pediatr 2006;165(9):654-7.

25. Toprak D, Soysal A, Torunoğlu MA, et al. PCR-based national bacterial meningitis surveillance in Turkey: years 2006 to 2009. Pediatr Infect Dis J 2014;33(10):1087-9.

26. Ceyhan M, Yildirim I, Sheppard CL, et al. Pneumococcal serotypes causing pediatric meningitis in Turkey: application of a new technology in the investigation of cases negative by conventional culture. Eur J Clin Microbiol Infect Dis 2010;29(3):289-93.

27. Özdemir H, Çiftçi E, Durmaz R, et al. Nasopharyngeal carriage of Streptococcus pneumoniae in healthy Turkish children after the addition of PCV7 to the national vaccine schedule. Eur J Pediatr 2014;173(3):313-20.

28. Ceyhan M,OzsurekciY,GürlerN, etal.Serotypedistribution of Streptococcus pneumoniae in children with invasive diseases in Turkey: 2008-2014. Hum Vaccin Immunother 2016;12(2):308-13. 